\title{
AN OBSERVATORY FOR MAPPING THE FAR UV DIFFUSE \\ GALACTIC EMISSION LINE BACKGROUND
}

\author{
F. L. ROESLER, J. HARLANDER and R. J. REYNOLDS \\ Dept. of Physics, University of Wisconsin, Madison, WI 5.3706
}

\begin{abstract}
A new instrumental concept for interference spectroscopy called Spatial Heterodyne Spectroscopy (SHS) is described. This instrument as currently demonstrated could provide important information on the structure, excitation, and dynamics of the $\simeq 10^{5} \mathrm{~K}$ component of the interstellar medium by providing velocity-resolved $\left(20 \mathrm{~km} \mathrm{~s}^{-1}\right)$ maps of the faint FUV emission line background over a hemisphere of the sky within a 5-6 year observation period. We are currently studying concepts expected to reduce this time by at least an order of magnitude.

In the SHS technique, an all-reflection dispersive interferometer produces a Fourier transform of the spectrum as two-dimensional spatial frequencies on an imaging detector. The system does not require scanning, and measures its own internal alignment state. Although the system suffers the conventional Fourier transform multiplex disadvantage associated with the photon noise in the background FUV continuum, we estimate that for a broad-band survey Spatial Heterodyne Spectroscopy as currently demonstrated can provide 4-5 fold gains over practical grating spectrometers of similar dimensions and spatial and spectral resolution. Field widened methods currently being studied promise additional gains of two orders of magnitude.
\end{abstract}

\section{Scientific Motivation}

The discovery of ultraviolet absorption lines (Jenkins 1978) and diffuse x-ray emission (Williamson et al. 1974) from highly ionized trace elements within the interstellar medium established the existence of a hot $\left(10^{5}-10^{6} \mathrm{~K}\right)$ component of the gas. This gas is generally believed to be produced by high velocity shocks from supernova remnants, expanding into the ambient ISM creating hot, low density bubbles, 50 to $200 \mathrm{pc}$ in radius. If long lived and frequent, these bubbles will profoundly influence the ISM structure by interconnecting and occupying most of the interstellar volume (Cox and Smith 1974; McKee and Ostriker 1977). Buoyant forces may carry the hot gas far above the Galactic midplane, producing a hot Galactic corona (Spitzer 1956) and large scale vertical circulations (Bregman 1980). While the existence of the hot component is firmly established by observations, its pervasiveness and influence on the structure of the interstellar medium has not yet been established.

The recent discovery of far-UV emission lines from hot $\left(10^{5} \mathrm{~K}\right)$ interstellar gas by Martin and Bowyer (1990) using a Rowland spectrograph on the Berkeley Extreme Ultraviolet/Far-Ultraviolet Shuttle Telescope has opened an important new window on the interstellar medium. The observations clearly show CIV $\lambda 1550$ and OIII $\lambda 1663$ emission lines from the diffuse interstellar medium with intensities typically 4500 photons $\mathrm{cm}^{-2} \mathrm{~s}^{-1} \mathrm{sr}^{-1}(0.06 R)$ and 2400 photons $\mathrm{cm}^{-2} \mathrm{~s}^{-1} \mathrm{sr}^{-1}$ $(0.03 R)$ respectively, at high Galactic latitudes. Also reported were detections at

Y. Kondo (ed.), Observatories in Earth Orbit and Beyond, 481-486.

(C) 1990 Kluwer Academic Publishers. Printed in The Netherlands. 
greater than $90 \%$ confidence of NIII $\lambda 1750$ and OIV plus SiIV $\lambda 1400$ with intensities of $1000-2000$ photons $\mathrm{cm}^{-2} \mathrm{~s}^{-1} \mathrm{sr}^{-1}(\simeq 0.02 R)$. A map over the sky of the intensities, radial velocities, and line profiles of these far-UV emission lines would provide important insights into the distribution and nature of hot interstellar gas. For example by comparison with $21 \mathrm{~cm}$ maps one could see whether the FUV occurred mainly inside the boundries of hot bubbles or on the outer surface of clouds immersed in a more widespread hot medium.

We are now developing a new instrumental concept for interference spectroscopy called Spatial Heterodyne Spectroscopy (SHS) (Harlander and Roesler 1990) that will provide the large throughputs needed to carry out such a survey. The instrument could be deployed as a dedicated compact observatory or as a part of a larger facility, preferably in far earth orbit or beyond to avoid the geocoronal photon haze. In the remainder of this paper we will describe the new concept and compare its projected performance with that of a conventional grating spectrometer of similar size and resolving power.

\section{The SHS}

We believe that SHS provides the first practical approach to interference spectroscopy in the far ultraviolet. The basic concept is most easily understood using the transmitting beamsplitter arrangement in Fig. 1 in which a conventional Michelson interferometer is shown with the return mirrors replaced by diffraction gratings $G_{1}$ and $G_{2}$. Light enters through aperture $A_{1}$ and is collimated by lens $L_{1}$. At the exit, lenses $L_{2}$ and $L_{3}$ relay the superposed coherent images of gratings $G_{1}$ and $G_{2}$ onto the image plane $I$ where a position sensitive detector records the Fizeau fringe pattern produced by the interferometer. The generation of Fizeau fringes of wavelength-dependent spatial frequency follows from the grating equation

$$
\sigma(\sin \theta+\sin (\theta-\gamma))=m / d
$$

where $\sigma$ is the wavenumber of light, $m$ is the order of diffraction, $\theta$ is the Littrow angle and $1 / d$ is the grating groove density. For an input point source of wavenumber $\sigma$, two coherent plane wavefronts are produced at the output (one from each arm) whose normals are inclined to the optical axis by angles $\gamma$ and $-\gamma$ respectively. Ignoring magnification by $L_{2}$ and $L_{3}$ these crossed wavefronts produce Fizeau fringes of spatial frequency

$$
f_{x}=2 \sigma \sin \gamma \simeq 4\left(\sigma-\sigma_{0}\right) \tan \theta
$$

where $\sigma_{0}$ is the Littrow $(\gamma=0)$ wavenumber. For input spectrum $B(\sigma)$, the intensity on the detector as a function of position $x$ is given by

$$
I(x)=\int_{0}^{\infty} B(\sigma)\left(1+\cos \left(2 \pi\left(4\left(\sigma-\sigma_{0}\right) x \tan \theta\right)\right)\right) d \sigma
$$

The Fourier transform of $I(x)$ will recover the input spectrum. No element has been mechanically scanned in this process. Zero spatial frequency does not correspond to zero wavenumber as in conventional FTS but to $\sigma=\sigma_{0}$. Effectively, 


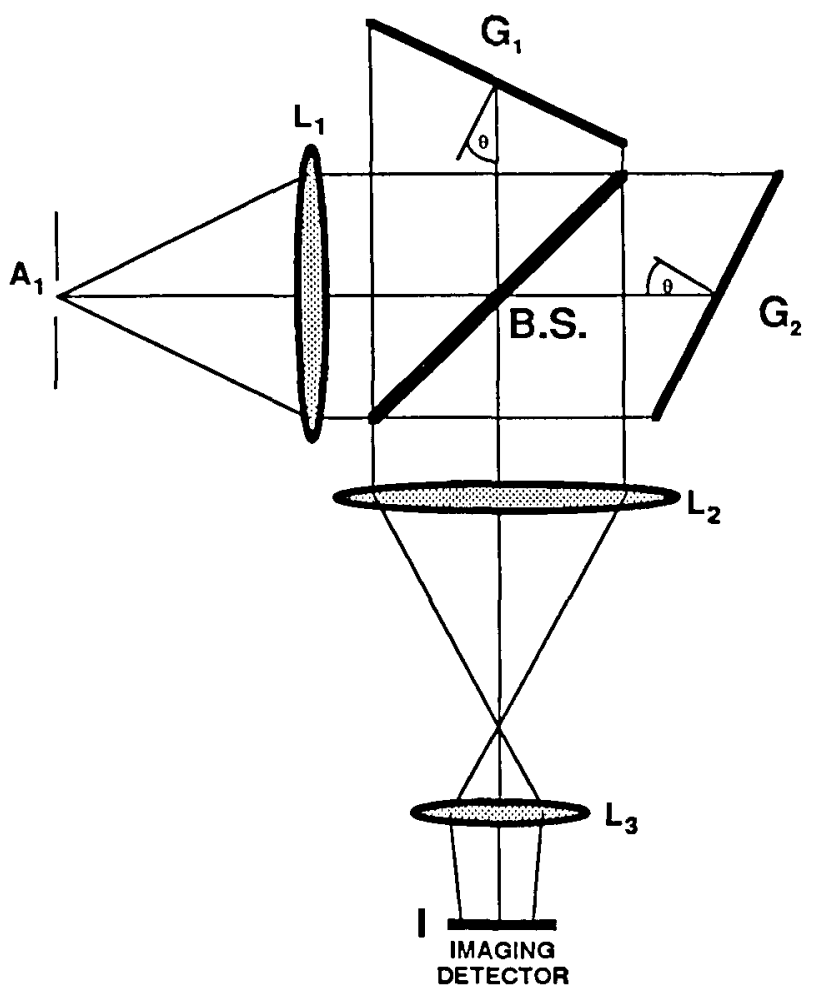

Fig. 1. Schematic diagram of the basic SHS configuration. Wavelength dependent Fizeau fringes produces by diffraction gratings $G_{1}$ and $G_{2}$ are recorded by a position sensitive detector $I$. The Fourier transform of the fringe pattern recovers the spectrum as described in the text.

SHS instruments record the entire path difference scanned by FTS simultaneously on a position sensitive detector without scanning, and heterodyne the interferogram with a frequency corresponding to the Littrow wavenumber of the gratings. For the geometry in Fig. 1, the limiting resolving power $(\sigma / \delta \sigma)$ is $R_{0}=4 W \sigma \sin \theta$ where $W$ is the width of the gratings. By including off axis input angles in the grating equation (Equation (1)), it can be shown that an SHS instrument achieves the same etendue (or throughput) as conventional FTS or Fabry-Perot interferometers. Optical tolerances are relaxed compared to conventional FTS because the Fizeau pattern from a monochromatic line can be used to correct phase errors in software.

An all-reflection version of SHS suitable for FUV operation can be achieved by replacing the transmitting beam splitter by the symmetrically blazed (or alternatively Holographic) grating $G_{0}$ as shown in Fig. 2. We have calculated and demonstrated in laboratory tests that instruments of this general design perform as predicted and achieve the high etendue characteristic of conventional interference spectrometers (Harlander and Roesler 1990). The resolving power of the system in Fig. 2 is $R_{0}=4 W \sigma \sin \theta\left(1-\tan \theta^{\prime} \cot \theta\right)$. 


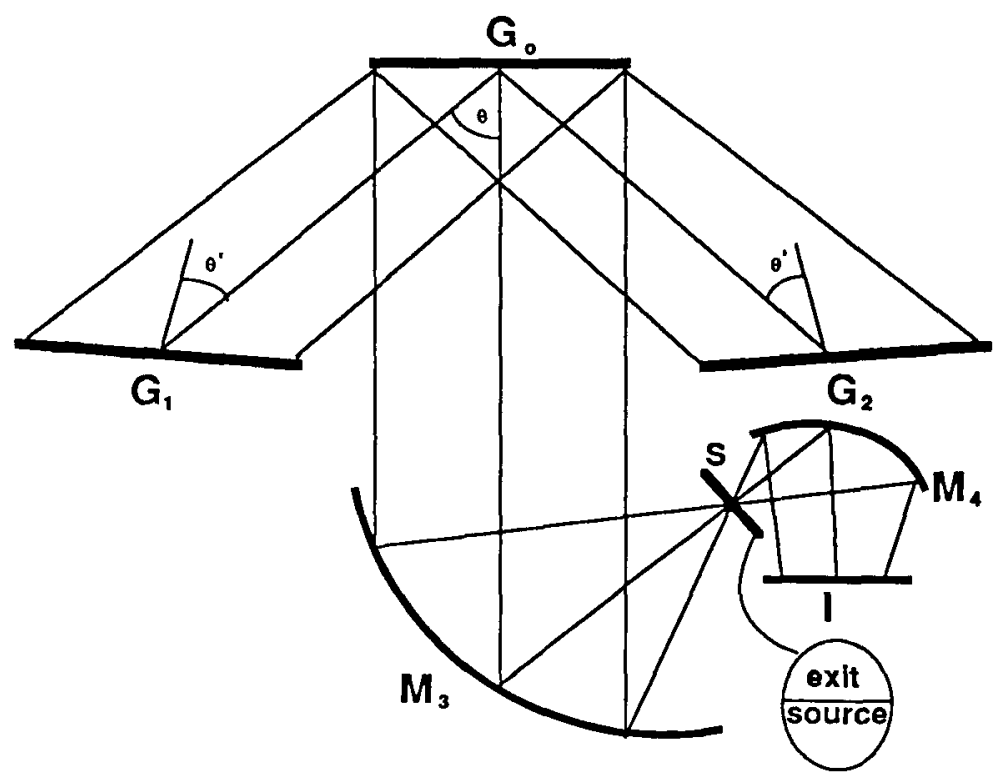

Fig. 2. Schematic diagram of the basic all-reflection SHS configuration. Light enters the system through the upper half of split aperture $S$ and exits through the lower half.

\section{Performance Prediction for a FUV Mapping Interferometer}

Figure 3 shows a concept suitable for comparing predicted SHS performance with that of a conventional grating spectrometer optimized for mapping intensities, velocities and line profiles of far UV emission lines. It is analogous to the configuration in Fig. 2, but has the advantage of separated input and output. Although is has an extra grating reflection its efficiency is comparable to the instrument in Fig. 2, which requires a reduced entrance aperture.

We have not had the means to test the configuration of Fig. 3, but ray tracing techniques verified on the other configurations indicate that its performance can be predicted with reasonable confidence. The comparison is complicated because SHS, like conventional FTS, is a true multiplexing system, and thus achieves a multiplex advantage when significant detector dark current is present, or a multiplex disadvantage when photon noise dominates.

For the comparison we have chosen echelle grating configurations (Harlander and Roesler, 1990, briefly discuss the SHS with echelle gratings) covering a $400 \AA$ range centered on $1500 \AA$. The conventional spectrometer uses a $30 \mathrm{~cm} \times 60 \mathrm{~cm}$ $63^{\circ}$ echelle grating and a $f / 2.5$ camera while the SHS system uses $30 \mathrm{~cm} \times 30 \mathrm{~cm}$ gratings symmetrically blazed at $30^{\circ}$, approximately. Both detectors are assumed to be $1000 \times 1000$ arrays of $20 \mu \mathrm{m}$ pixels. We have used mirror efficiencies of 0.8 , grating efficiencies of 0.4 and detector $\mathrm{QE}$ of 0.2 . Lyman alpha has been excluded by a $\mathrm{CaF}_{2}$ because of the large photon noise that it could introduce in the multiplexing SHS system especially in near-earth orbit. Full multiplex disadvantage is 


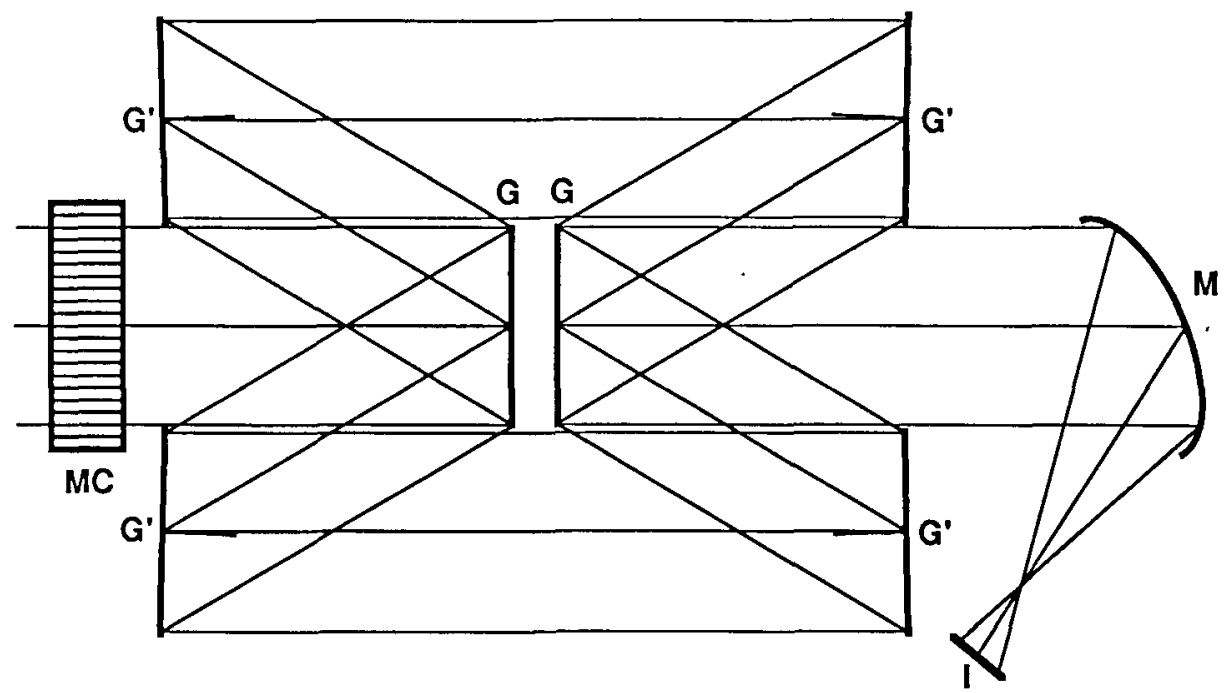

Fig. 3. SHS system used for comparison with an echelle spectrograph. Light enters the system through a mechanical collimator MC. Gratings $G$ and $G^{\prime}$ have slightly different groove densities so as to produce the required dispersion while maintaining a relatively compact size.

assumed although non-negligible detector noise might be present in the required long exposure times. A single measurement reaching $S N=10$ for a $.025 R$ line in a continuous background of 400 photon $\mathrm{cm}^{-2} \mathrm{~s}^{-1} \mathrm{sr}^{-1} \AA^{-1}$ is estimated to take about 90 min with the SHS system and about $400 \mathrm{~min}$ with the grating system. Thus SHS has an advantage between 4 and 5 .

A survey with $1^{\circ}$ angular resolution and $20 \mathrm{kms}^{-1}$ radial velocity resolution, providing $5-10 \sigma$ detections of the observed CIV and OIII emission is estimated to take 5-6 years with a modest size ( $\simeq 30 \mathrm{~cm}$ diameter optics) SHS instrument. By binning into larger angular resolution elements, fainter lines such as NIII $\lambda 1750$, OIV and SiIV $\lambda 1400$, and NIV $\lambda 1490$ might be measured.

We are also studying field-widened methods for SHS systems. We have calculated gains ¡beyond $i$ that of simple $S_{H S}$ of $\simeq 4$ for an all-reflection system not yet optimized for minimum aberrations, and are confident that further refinement will push the predicted gains well over an order of magnitude. For a system using a thin transmitting wedge of $\mathrm{MgF}_{2}$ or $\mathrm{CaF}_{2}$, additional gains of more than two orders of magnitude appear practical.

We believe that SHS can have an important impact on planned and future NASA programs, and should be vigorously developed to allow timely decisions on its place in the mainstream of space observatory instrumentation for the next decade and the next century of space exploration. 


\section{References}

Bergman, J.N.: 1980, Ap.J. 236, 577

Cox, D.P. and B.W. Smith: 1974, Ap. J. (Letters) 189, L105

Harlander, J. and F. L. Roesler: 1990, SPIE 1235, in Press

Jenkins, E.B.: 1978, Ap. J. 219, 845

Martin, C. and S. Bowger: 1990, Ap. J. 350, 242

McKee, C.F. and J.P. Ostriker: 1977, Ap. J. 218, 148

Spitzer, L., Jr.: 1956, Ap. J. 124, 20

Williamson, F.O., W.T. Sanders, W.L. Kraushaar, D. McCammon, R. Borken, and A.N.

Bunner: 1974, Ap. J. (Letters) 193, L133 